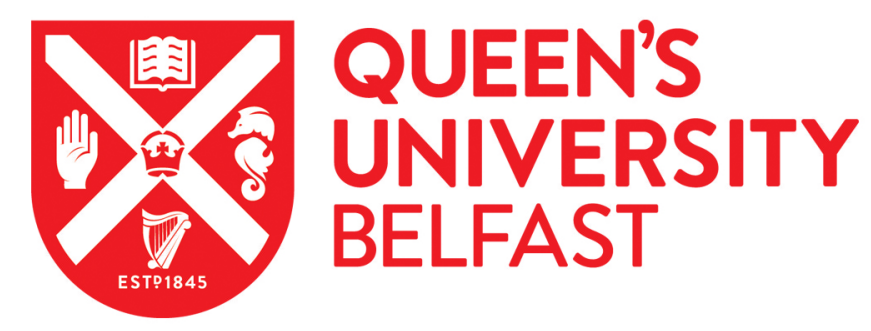

\title{
Putting proprioception for balance to the test: Contrasting and combining sway referencing and tendon vibration
}

Doumas, M., Valkanidis, T. C., \& Hatzitaki, V. (2018). Putting proprioception for balance to the test: Contrasting and combining sway referencing and tendon vibration. Gait and Posture.

https://doi.org/10.1016/j.gaitpost.2018.10.012

Published in:

Gait and Posture

Document Version:

Peer reviewed version

Queen's University Belfast - Research Portal:

Link to publication record in Queen's University Belfast Research Portal

\section{Publisher rights}

Copyright 2018 Elsevier.

This manuscript is distributed under a Creative Commons Attribution-NonCommercial-NoDerivs License

(https://creativecommons.org/licenses/by-nc-nd/4.0/), which permits distribution and reproduction for non-commercial purposes, provided the author and source are cited.

\section{General rights}

Copyright for the publications made accessible via the Queen's University Belfast Research Portal is retained by the author(s) and / or other copyright owners and it is a condition of accessing these publications that users recognise and abide by the legal requirements associated with these rights.

Take down policy

The Research Portal is Queen's institutional repository that provides access to Queen's research output. Every effort has been made to ensure that content in the Research Portal does not infringe any person's rights, or applicable UK laws. If you discover content in the Research Portal that you believe breaches copyright or violates any law, please contact openaccess@qub.ac.uk. 
In press at Gait and posture

Putting proprioception for balance to the test: Contrasting and combining sway referencing and tendon vibration

Michail Doumas ${ }^{1}$, Theofilos Ch. Valkanidis ${ }^{1}$ and Vassilia Hatzitaki ${ }^{2}$ ${ }^{1}$ School of Psychology, Queen's University Belfast, Belfast, UK

${ }^{2}$ Laboratory of Motor Behavior and Adapted Physical Activity, School of Physical Education and Sport Sciences, Aristotle University of Thessaloniki, Thessaloniki, Greece

Word count: 3000

Address correspondence to Michail Doumas, School of Psychology, Queen's University Belfast, 18-30 Malone Road, Belfast, BT9 5BN, UK. Email: m.doumas@qub.ac.uk, Tel: +44(0)289097 4605, Fax: +44 (0)2890975486.

Keywords: Postural control; balance; proprioception; tendon vibration; sway reference; sensory reweighting. 


\begin{abstract}
Background: Postural control relies on sensory information from visual, vestibular and proprioceptive channels, with proprioception being the key sensory modality in this task. Two well-established ways of manipulating proprioceptive information in postural control are tendon vibration and sway referencing. The aim of the present study was to assess postural adaptation when inaccurate proprioceptive information is introduced using tendon vibration and sway referencing in isolation and combination.
\end{abstract}

Methods: Seventeen young adults were asked to stand, without vision, for 2 minutes on a fixed surface (Baseline) immediately followed by 3 minutes of bilateral Achilles tendon vibration, sway reference, or combined presentation of the two manipulations (Adaptation) and finally 3 minutes of standing on a fixed surface (Aftereffect).

Results: During adaptation, vibration showed the lowest sway variability, followed by sway reference and the combined condition. Spectral analyses focusing on the dominant frequencies in this task $(0-0.4 \mathrm{~Hz})$ showed that in the first half of adaptation sway amplitude was greater when the two manipulations were combined compared with each manipulation alone. However, in the second half differences between sway reference and the combined condition disappeared but differences between vibration and the other two conditions increased.

Conclusion: We interpret these findings primarily as due to a prolonged attenuation in effects of vibration over the course of the adaptation phase and we offer two explanations for this phenomenon. One is a decline in neurotransmitter release from the group Ia terminals and the other is sensory reweighting which down-weights proprioception and up-weights the accurate, vestibular information. 


\section{Introduction}

Postural control is achieved using sensory information from visual, vestibular and proprioceptive channels. This information is integrated by an adaptive sensory reweighting process which weights each channel based on its relative reliability [1] in order to produce the appropriate muscle torque to maintain stability [2]. During standing on fixed and slightly moving surfaces, the proprioceptive channel is weighted the highest, followed by the visual and the vestibular channels $(50 \%, 35 \%$ and $15 \%$ respectively) $[2,3]$. The nature of proprioceptive contribution to postural control can be studied using two well-established, but markedly different methods of inducing inaccurate proprioceptive information, tendon vibration and support-surface sway reference.

Tendon vibration applied at $80-100 \mathrm{~Hz}$ activates Ia spindle afferents, triggering the tonic vibration reflex and creating the illusion that the muscle is lengthening [4-7],. While standing, vibration is typically applied bilaterally over the Achilles tendons and results in an illusory lengthening of dorsi-flexors, which would normally signal forward body sway. This illusory lengthening is followed by activation of the vibrated muscles and movement in the opposite direction, i.e. a backward body lean.

Support surface sway referencing [8] is also used to manipulate proprioceptive information for postural control. When standing on a fixed surface, naturally occurring body sway causes a change in ankle angle, and in turn, in the length of muscles and tendons around the ankle joint. These changes activate Ia afferents which provide proprioceptive information for maintaining upright stance. When sway referencing is introduced, the support surface is tilted in direct proportion to body sway and as a result ankle angle is kept near constant [8-11]. Thus, in contrast to tendon vibration, 
sway referencing affects proprioceptive information about body sway without interfering directly with proprioceptive receptors, and even though proprioceptive information per se is intact, proprioceptive information about body sway is near eliminated $[9,12]$.

Both manipulations increase postural sway relative to baseline, followed by a gradual decrease over time both in sway referencing $[10,13,14]$ and in tendon vibration but in a longer time scale, i.e. across trials or over days[15]. This reduction has been attributed to down-weighting of the inaccurate proprioceptive information and upweighting of accurate visual and vestibular information over time [2,9]. Given that both manipulations are subject to the same sensory reweighting process, it is reasonable to predict that introducing them simultaneously would cause an additive increase in postural sway. However, previous evidence suggests that effects of tendon vibration are attenuated when the support surface is unstable [15-19]. The reasons for this attenuation and the time course of adaptation have not been systematically studied. For instance, one study that contrasted postural adaptation during vibration while standing on a sway referenced surface showed no sway reduction over nine successive 20 s trials in a single day but a reduction in sway over three days [15].

The aim of the present study was to compare the time course of postural adaptation when tendon vibration or sway referencing is introduced alone, with the simultaneous application of both manipulations. We used a single, long (3min) adaptation phase in order to study the time course of adaptation continuously, rather than in successive short trials like in previous studies $[15,20]$. We predicted that this paradigm would better capture the gradual postural adaptation process to the isolated or combined manipulations and reveal whether the attenuated effects of vibration 
observed previously when standing on unstable surfaces [15-19] are immediate or last longer over the course of adaptation.

\section{Methods}

\subsection{Participants}

Seventeen adults, 4 males and 13 females $\left(\mathrm{M}_{\text {age }}=26.12, \mathrm{SD}_{\text {age }}=4.6 y e a r s\right)$ participated in this study. Participants reported no major neurological or musculoskeletal disorders, no intake of medication that affects postural control and no dizziness or vestibular problems. They provided written informed consent and the research protocol was approved by the School of Psychology, Queen's University Belfast research ethics committee.

\subsection{Apparatus}

Tendon vibration (Figure 1, top) was bilaterally applied using two vibrators (TechnoConcept $\AA$ VB 115, frequency: $80 \mathrm{~Hz}$, amplitude: $3 \mathrm{~mm}$ ) attached to the Achilles tendons using rubber bands. They were turned on and off via computer-generated signals triggered using a CED Micro1401 data acquisition card.

Sway Referencing was implemented using a Neurocom Smart Balance Master (Natus inc.) comprising 18" by 18" dual force plates which recorded vertical forces (frequency: 100Hz). These forces were used to derive Center of Pressure (COP) trajectories in the Anterior-Posterior (AP) and Medio-Lateral (ML) directions. COP was low-pass filtered to estimate Centre of Mass trajectories which in turn were used to drive a servo-controlled motor that tilted the surface in the sagittal plane (toes-down 
and toes-up) in direct proportion to body sway (gain=1). For example, when the body swayed $1^{\circ}$ forward the platform tilted $1^{\circ}$ toes-down thereby keeping ankle angle nearly unchanged $[8,11,21]$.

Movement kinematics in three dimensions were recorded at $100 \mathrm{~Hz}$ using a Codamotion CX1 sensor unit (Charnwood Dynamics Ltd., Rothley, Leicestershire, UK), placed approximately 2 meters behind the participant. Markers were placed on the C7 and L5 vertebra, on the right and left superior calcanei as well as on the force plates' surface.

\subsection{Tasks and Procedure}

The experiment took place in a single session. Participants filled out a medical questionnaire and then the experimenter described the study's protocol. Participants were asked to stand barefoot on the dual force platform wearing a safety harness and blindfold, at a stance width determined by the system's manufacturer, and to try to maintain balance. Prior to testing, participants familiarized themselves with the three manipulations by performing 1-2 10s trials in each one.

The main experiment comprised three 8-minute conditions (Figure 1) the order of which was counterbalanced across participants. In the 'Vibration' condition (Figure 1, top), participants were asked to stand for $2 \mathrm{~min}$ on a fixed surface to establish a baseline, followed by $3 \mathrm{~min}$ of adaptation to bilateral tendon vibration and then 3 minutes on a fixed surface to assess possible aftereffects. In the 'Sway Referenced' condition (Figure 1, middle), participants were introduced to a sway referenced surface during adaptation maintaining the other two phases (baseline, aftereffect) the same. Finally, in the 
'Combined' condition the same paradigm was used with both sway reference and tendon vibration manipulations presented simultaneously during adaptation.

\subsection{Data analysis}

Position-time functions from the C7 marker, ankle and moving platform markers were low-pass filtered at $4 \mathrm{~Hz}$ using a $5^{\text {th }}$ order Butterworth dual-pass filter. Then, C7 tilt angles over time were calculated in the AP direction using kinematic information from ankle and C7 markers. C7 tilt angle information was used to calculate C7 angular velocity variability (SD) in 24 successive 20 s windows for each condition. Spectral analyses were performed in the first (5-90s) and second half (90-175s) of the adaptation phase using discrete Fourier transform. This analysis yielded the amplitudefrequency spectrum in bins of $0.0118 \mathrm{~Hz}$. Amplitude at frequencies up to $0.4 \mathrm{~Hz}$ (34 frequencies) was calculated as this is the frequency range with the highest amplitude in this task. Then, amplitude was averaged in 4 frequency bins: $0.0118-0.0941 \mathrm{~Hz}, 0.1059$ $0.2 \mathrm{~Hz}, 0.2117-0.0 .2941 \mathrm{~Hz}$ and $0.3058-0.4 \mathrm{~Hz}$. We refer to these bins as $0-0.1,0.1-0.2$, 0.2-0.3 and $0.3-0.4 \mathrm{~Hz}$ for simplicity. Data processing was performed using Matlab (The Mathworks, Natick, MA, USA) and statistical analyses using JASP (Version 0.8.5). Statistical analyses were conducted using within-subjects repeated measures analyses of variance. In cases of sphericity violation a Huynh-Feldt correction was applied.

\section{Results}

Figure 2 depicts C7 tilt angle trajectories for the three conditions. In the Vibration condition (Figure 2, top), vibration induced the well-documented backward lean, 
followed by sway reduction over time. Sway Reference (Figure 2, middle) caused slow (0.1-0.2Hz) body oscillations[9,12]. Sway amplitude decreased over time and returned to baseline levels when the fixed platform was restored. In the Combined condition (Figure 2, bottom), both the backward lean and the slow oscillations were observed during adaptation. When the manipulations stopped the oscillation disappeared and the body returned to its initial position.

\subsection{C7 angular velocity $S D$}

Baseline. C7 angular velocity SD (Figure 3) at baseline was assessed using a 3 by 6 repeated-measures ANOVA with factors Condition (Vibration, Sway Reference and Combined) and Window (1-6). Results showed a main effect of Condition $F(2,32)=$ 3.42, $\mathrm{p}=.045, \eta 2=.18$. Pair-wise post-hoc t-tests with Bonferroni correction showed that neither contrast was significant (all ps>.05). No other significant effects were shown.

Adaptation. A 3 (Condition) by 9 (Window) repeated-measures ANOVA revealed that velocity SD decreased over time $F(5.61,89.81)=10.61, \mathrm{p}<.001, \eta 2=.4$. Furthermore, velocity SD was different among conditions $F(2,32)=32.821, p<.001, \eta 2=.67$. Bonferroni corrected pairwise comparisons $(\alpha=0.017)$ showed lower velocity SD in the Vibration compared with Sway Reference $\mathrm{t}(16)=5.13, \mathrm{p}<.001$ and Combined condition $t(16)=7.99, p<.001$ but the difference between Sway Reference and the Combined condition $\mathrm{t}(16)=2.86, \mathrm{p}=.022$ did not reach significance. Similarly, the Condition by Window interaction did not reach significance $F(4.94,79.01)=1.69, p=.01$. These results show that the primary difference was between Vibration and the two other conditions. Visual inspection of Figure 3 suggests that this difference was more 
pronounced in the second half of the adaptation phase. To assess this pattern we performed exploratory pair-wise t-tests in each adaptation window between the Combined condition and the other two conditions adjusted for multiple comparisons (18 comparisons, $\alpha=.003$ ). Results showed that Vibration was different from Combined in windows 7 and 9-15 $(\mathrm{t}(16)=5.74-3.6, \mathrm{p}<.003)$ but no differences were observed between the Sway Reference and Combined conditions.

Aftereffect. To assess the presence and the duration of an aftereffect in velocity SD, we performed separate repeated-measures ANOVAs for each condition, applying planned contrasts between the average velocity SD at baseline with each window after removal of the manipulation (windows 16-24). For Vibration, results showed greater SD from baseline $(\mathrm{M}=0.32, \mathrm{SD}=0.11 \mathrm{deg} / \mathrm{s})$, at window $16 \mathrm{t}(16)=15.98, \mathrm{p}<.001$ but lower $\mathrm{SD}$ in windows 22-24 (22: $\mathrm{t}(16)=2.55, \mathrm{p}=.012 ; 23: \mathrm{t}(16)=2.89, \mathrm{p}=.004 ; 24: \mathrm{t}(16)=3.14$, $p=.002)$. For Sway Reference, velocity SD was greater than baseline $(M=0.38, S D=0.16$ $\mathrm{deg} / \mathrm{s}$ ) in windows $16 \mathrm{t}(16)=10.1, \mathrm{p}<.001$, but lower than baseline average in windows $21 \mathrm{t}(16)=2.1, \mathrm{p}=.038$ and $24 \mathrm{t}(16)=2.05, \mathrm{p}=.042$. Finally, in the Combined condition, velocity SD was greater than baseline $(M=0.42, S D=0.24 \mathrm{deg} / \mathrm{s})$ in windows 16 $t(16)=13.206, p<.001$, but lower in windows $20 \mathrm{t}(16)=2.48, \mathrm{p}=.010$ and $23 \mathrm{t}(16)=2.61$, $\mathrm{p}=.010$

\subsection{Spectral analysis}

In order to contrast differences in adaptation in the three conditions in the frequency domain, we performed a 3 (Condition) by 4 (Frequency) by 2 (Half) repeated measures ANOVA for spectral amplitude (Figure 4). Results showed a main effect of Condition $F(2,32)=61.53 P<.001, \eta^{2}=.78$, a marginally significant main effect of Half reflecting a 
reduction in amplitude from the first to the second half of adaptation $F(1,32)=4.16$, $\mathrm{p}=.054, \eta^{2}=.29$ and a Condition by Half interaction $\mathrm{F}(2,32)=5.6, \mathrm{P}<.05, \eta^{2}=.26$. Pair wise t-tests adjusted for multiple comparisons $(\alpha=.006)$ revealed that in the first half, the Combined condition exhibited higher amplitude than Sway Reference $t(16)=7.54$, $\mathrm{p}<.001$ and Vibration $\mathrm{t}(16)=3.14, \mathrm{p}=.006$ and that Sway Reference showed higher amplitude than Vibration $\mathrm{t}(16)=8.22, \mathrm{p}<.001$. In the second half, differences between Combined and Sway Reference disappeared but Vibration still had lower amplitude than both (Sway reference $t(16)=7.29, \mathrm{p}<.001$, Combined $t(16)=8.36, \mathrm{p}<.001)$. The change in pattern between the first and second half (Figure 4) can be explained by the amplitude reduction which was significant in the Combined $(\mathrm{p}=.007)$ and Vibration $(p=.019)$ conditions but not evident in Sway Reference. Sway amplitude was also reduced across the 4 frequency bands $F(1.56,24.96)=109.112 \mathrm{P}<.001 \eta^{2}=.87$ (Figure 4). Also , the effect of condition was larger in lower frequencies, as shown by frequency by condition $F(2.62,41.86)=13.13, \mathrm{p}<.001, \eta^{2}=.45, \mathrm{p}=.004, \eta^{2}=.16$ and frequency by condition by half interactions $F(2.52,40.31)=3.12, p=.044, \eta^{2}=.16$.

\section{Discussion}

The aim of the present study was to assess postural adaptation when inaccurate proprioceptive information is introduced by means of tendon vibration and support surface sway referencing in isolation and combination. Results showed that during adaptation sway variability was greater in the combined and sway reference conditions compared to vibration but the greater variability in the combined compared with the sway reference conditions only approached significance. Spectral analysis contrasting the first with the second part of adaptation showed that in the first half vibration 
showed the lowest amplitude, followed by sway reference and then the combined condition. However, in the second half differences between sway reference and the combined condition disappeared but differences between vibration and the combined condition increased. We interpret these findings primarily as due to an attenuation in effects of vibration over the course of adaptation.

Vibration induced an increase in sway variability compared to baseline but this was smaller than the increase induced by sway referencing, and in turn sway variability in sway referencing was smaller than variability in the combined condition. However, the pattern of differences across the three conditions changed over the course of adaptation with sway variability being significantly lower in the vibration than the combined condition in window 7 and across windows 9 to 15 (over the last two minutes of adaptation). On the other hand, there were no differences in sway variability between the sway reference and combined conditions. This suggests that most of the variability induced in the combined condition originated from sway referencing while the effect of vibration was inhibited. The attenuation of vibration effects when standing on an unstable support is well-documented and has been attributed to sensory reweighting processes[15-17,22]. Specifically, standing on an unstable support (e.g. foam) decreases reliance on muscle spindle afferent input and increases the vestibular contribution to the control of balance[23]. This is accomplished via vestibulospinal projections onto post activation depression interneurons [24], which increase the pre-synaptic inhibition of the Ia afferent input from the muscle to the motor neuron pool. Thus, presynaptic depression of the segmental Ia afferent inputs onto spinal motor neurones reduces segmental reflexes without modulating the inputs projecting to supraspinal levels $[25,26]$. This indicates a shift towards greater supraspinal control when balance is 
compromised (i.e. by sway reference) while inhibiting spinal reflexes induced by tendon vibration[27].

In order to specifically investigate how the two proprioceptive manipulations are modulated over periods of time longer than in previous research $(>30 \mathrm{~s})$, we compared spectral sway amplitude $(0-0.4 \mathrm{~Hz})$ between the $1^{\text {st }}(5-120 \mathrm{~ms})$ and $2^{\text {nd }}(120$ $175 \mathrm{~ms}$ ) half of the 180s adaptation period. Spectral analysis confirmed that sway amplitude decreased between the $1^{\text {st }}$ and $2^{\text {nd }}$ half primarily in the combined condition, less so in the vibration condition and not at all in the sway reference condition. Furthermore, the difference in sway variability between sway referencing and the combined condition was small during the $1^{\text {st }}$ half of adaptation and no longer present during the $2^{\text {nd }}$ half. At the same time, the vibration-induced sway variability continued to decrease over the $2^{\text {nd }}$ half (between time windows 11-15) increasing the difference between vibration and the two other conditions. Taken together, these results suggest that the attenuation of vibration when standing on an unstable support is not immediate as previously suggested but extends over longer adaptation periods (wanes over the entire 180s phase). This continuous attenuation of the vibration-induced effects on sway variability may have two possible explanations.

One explanation could be that it is due to neurotransmitter depletion in the Ia afferent feedback loop. Prolonged vibration (>30s) results in decreased discharge sensitivity of muscle spindles [28], decreased maximal voluntary force production[29,30], decreased force fluctuations during submaximal tasks and inhibition of the H-reflex of the ankle muscles[31]. These effects have been attributed to a decline in neurotransmitter release from the group Ia terminals[32]. Another explanation is that this effect is due to the gradual down-weighting of the inaccurate proprioceptive channel over time while increasing reliance to the accurate, vestibular channel. In 
contrast to our findings, however, Dettmer et al.[15] using combined sway referencing with tendon vibration, did not observe any attenuation of the vibration effects on postural stability across nine short (20s) trials followed by $5 \mathrm{~s}$ breaks performed in a single experimental session. This was probably due to the intermittent nature of tendon vibration in that study and supports our idea that the attenuation of vibration effects observed in the present study over longer timescales requires constant, rather than intermittent stimulation. This intermittent stimulation may have prevented neurotransmitter depletion and may have allowed for (partial) restoration of sensory channels' weights by being exposed to the veridical environment for $5 \mathrm{~s}$. On the other hand, vibration effects were attenuated across successive days of repeated exposure, an adaptation that was attributed to the down-weighting of the less reliable proprioceptive inputs. Taken together, these findings suggest that the attenuation of the vibrationinduced sway observed over the later part of adaptation in the present study is probably related to the decreased sensitivity of the Ia afferent feedback loop as a result of the prolonged vibration ( $>30$ s) while it is less likely to be the result of reweighting or postural strategy changes.

Aftereffects were similar in duration among all three conditions with sway variability returning to baseline levels within the first $20 \mathrm{~s}$ after the end of adaptation. This confirms that reweighing of inaccurate proprioceptive information is independent of the type of proprioceptive manipulation probably because this is modulated at the higher levels of the CNS.

A possible limitation of our study is that we applied vibration in one tendon on one side of the ankle joint, which may allow the other, non-vibrated side to compensate for the induced inaccurate proprioceptive information. A more complete design might have been to co-vibrate the two sides, however, studies using this method have shown 
that neither a kinesthetic illusion nor a motor response at the joint is evoked [33,34] and concluded that illusory perception of movement is elicited only when there is difference in proprioceptive signals between two sides. Nevertheless, within-channel proprioceptive reweighting might be possible across different joints and this idea deserves further consideration by future studies.

In conclusion, the results of the present study confirmed that the effects of tendon vibration are attenuated when standing on a sway referencing support. More importantly, this study showed for the first time that this attenuation is a dynamic process that results in further attenuation over time probably due to a gradually developing decreased sensitivity of the segmental reflex loop. 


\section{Acknowledgements}

The authors would like to thank Haralampos Sotirakis and Adam Goodworth for their help with data analysis.

\section{Conflict of interest statement}

The authors have no conflict of interest to declare 


\section{References}

[1] M.O. Ernst, M.S. Banks, Humans integrate visual and haptic information in a statistically optimal fashion, Nature. 415 (2002) 429-433. doi:10.1038/415429a.

[2] R.J. Peterka, Sensorimotor integration in human postural control, J Neurophysiol. 88 (2002) 1097-1118. doi:Doi 10.1152/Jn.00605.2001.

[3] M. Cenciarini, R.J. Peterka, M. Cenciarini, R.J. Peterka, Stimulus-Dependent Changes in the Vestibular Contribution to Human Postural Control StimulusDependent Changes in the Vestibular Contribution to Human Postural Control, (2006) 2733-2750. doi:10.1152/jn.00856.2004.

[4] R. Bianconi, J.P. van der Meulen, The response to vibration of the end organs of mammalian muscle spindles., J. Neurophysiol. 26 (1963) 177-90.

[5] D. Burke, K.E. Hagbarth, L. Lofstedt, B.G. Wallin, The responses of human muscle spindle endings to vibration during isometric contraction, J. Physiol. 261 (1976) 695-711. doi:10.1113/jphysiol.1976.sp011580.

[6] J.P. Roll, J.P. Vedel, Kinaesthetic role of muscle afferents in man, studied by tendon vibration and microneurography, Exp. Brain Res. 47 (1982) 177-190. doi:10.1007/BF00239377.

[7] J.P. Roll, J.P. Vedel, E. Ribot, Alteration of proprioceptive messages induced by tendon vibration in man: a microneurographic study, Exp. Brain Res. 76 (1989) 213-222. doi:10.1007/BF00253639.

[8] L.M. Nashner, F.O. Black, C. Wall 3rd, Adaptation to altered support and visual conditions during stance: patients with vestibular deficits, J Neurosci. 2 (1982) 536-544. http://www.ncbi.nlm.nih.gov/pubmed/6978930.

[9] R.J. Peterka, P.J. Loughlin, Dynamic regulation of sensorimotor integration in human postural control, J Neurophysiol. 91 (2004) 410-423.

[10] M. Doumas, R.T. Krampe, Adaptation and reintegration of proprioceptive information in young and older adults' postural control., J. Neurophysiol. 104 (2010) 1969-77. doi:10.1152/jn.00345.2010.

[11] S. Clark, M.A. Riley, Multisensory information for postural control: swayreferencing gain shapes center of pressure variability and temporal dynamics, Exp Brain Res. 176 (2007) 299-310. doi:10.1007/s00221-006-0620-6.

[12] M. Doumas, R.T. Krampe, Adaptation and reintegration of proprioceptive information in young and older adults' postural control, J. Neurophysiol. 104 (2010). doi:10.1152/jn.00345.2010.

[13] C.E. Craig, G.H.M. Calvert, M. Doumas, Effects of the availability of accurate proprioceptive information on older adults' postural sway and muscle cocontraction, Eur. J. Neurosci. 46 (2017). doi:10.1111/ejn.13703.

[14] C.E. Craig, D.J. Goble, M. Doumas, Proprioceptive acuity predicts muscle cocontraction of the tibialis anterior and gastrocnemius medialis in older adults' 
dynamic postural control, Neuroscience. 322 (2016). doi:10.1016/j.neuroscience.2016.02.036.

[15] M. Dettmer, A. Pourmoghaddam, D.P. O'Connor, C.S. Layne, Interaction of support surface stability and Achilles tendon vibration during a postural adaptation task, Hum. Mov. Sci. 32 (2013) 214-227. doi:10.1016/j.humov.2012.12.002.

[16] V. Hatzitaki, M. Pavlou, A.M. Bronstein, The integration of multiple proprioceptive information: effect of ankle tendon vibration on postural responses to platform tilt, Exp. Brain Res. 154 (2004) 345-354. doi:10.1007/s00221-003-1661-8.

[17] Y.P. Ivanenko, Support stability influences postural responses to muscle vibration in humans, Eur. J. Neurosci. 11 (1999) 647-654. doi:10.1046/j.14609568.1999.00471.x.

[18] Y.P. Ivanenko, I.A. Solopova, Y.S. Levik, The direction of postural instability affects postural reactions to ankle muscle vibration in humans, Neurosci. Lett. 292 (2000) 103-106. doi:10.1016/S0304-3940(00)01438-5.

[19] H. Kiers, S. Brumagne, J. van Dieën, P. van der Wees, L. Vanhees, Ankle proprioception is not targeted by exercises on an unstable surface, Eur. J. Appl. Physiol. 112 (2012) 1577-1585. doi:10.1007/s00421-011-2124-8.

[20] M.J.E. Turnock, C.S. Layne, Variations in Linear and Nonlinear Postural Measurements under Achilles Tendon Vibration and Unstable Support-Surface Conditions, 42 (2010) 61-69.

[21] R.J. Peterka, F.O. Black, Age-related changes in human posture control: sensory organization tests, J Vestib Res. 1 (1990) 73-85.

http://www.ncbi.nlm.nih.gov/pubmed/1670139.

[22] S. Baudry, J. Duchateau, Age-related influence of vision and proprioception on Ia presynaptic inhibition in soleus muscle during upright stance, J. Physiol. 590 (2012) 5541-5554. doi:10.1113/jphysiol.2012.228932.

[23] R. Fitzpatrick, D.K. Rogers, D.I. McCloskey, Stable human standing with lowerlimb muscle afferents providing the only sensory input., J. Physiol. 480 (1994) 395-403.

[24] J.F. Iles, J. V Pisini, Cortical modulation of transmission in spinal reflex pathways of man., J. Physiol. 455 (1992) 425-446. doi:10.1113/jphysiol.1992.sp019309.

[25] L.M. Nashner, Adapting reflexes controlling the human posture, Exp Brain Res. 26 (1976) 59-72. http://www.ncbi.nlm.nih.gov/pubmed/964327.

[26] P. Rudomin, R.F. Schmidt, Presynaptic inhibition in the vertebrate spinal cord revisited, Exp. Brain Res. 129 (1999) 1-37. doi:10.1007/s002210050933.

[27] W.E. McIlroy, D.C. Bishop, W.R. Staines, A.J. Nelson, B.E. Maki, J.D. Brooke, Modulation of afferent inflow during the control of balancing tasks using the lower limbs, Brain Res. 961 (2003) 73-80. doi:https://doi.org/10.1016/S00068993(02)03845-3. 
[28] E. Ribot-Ciscar, C. Rossi-Durand, J.-P. Roll, Muscle spindle activity following muscle tendon vibration in man, Neurosci. Lett. 258 (1998) 147-150. doi:https://doi.org/10.1016/S0304-3940(98)00732-0.

[29] L.G. Bongiovanni, K.E. Hagbarth, L. Stjernberg, Prolonged muscle vibration reducing motor output in maximal voluntary contractions in man., J. Physiol. 423 (1990) 15-26. doi:10.1113/jphysiol.1990.sp018008.

[30] L.G. Bongiovanni, K.E. Hagbarth, Tonic vibration reflexes elicited during fatigue from maximal voluntary contractions in man., J. Physiol. 423 (1990) 1-14. doi:10.1113/jphysiol.1990.sp018007.

[31] A. Van Boxtel, Differential effects of low-frequency depression, vibration-induced inhibition, and posttetanic potentiation on H-reflexes and tendon jerks in the human soleus muscle., J. Neurophysiol. 55 (1986) 551-568.

[32] J. Ushiyama, K. Masani, M. Kouzaki, H. Kanehisa, T. Fukunaga, Difference in aftereffects following prolonged Achilles tendon vibration on muscle activity during maximal voluntary contraction among plantar flexor synergists, J. Appl. Physiol. 98 (2005) 1427-1433. doi:10.1152/japplphysiol.00613.2004.

[33] E. Shibata, F. Kaneko, M. Katayose, Muscular responses appear to be associated with existence of kinesthetic perception during combination of tendon covibration and motor imagery, Exp. Brain Res. 235 (2017) 3417-3425. doi:10.1007/s00221-017-5057-6.

[34] J.C. Gilhodes, J.P. Roll, M.F. Tardy-Gervet, Perceptual and motor effects of agonistantagonist muscle vibration in man, Exp. Brain Res. 61 (1986) 395-402. doi:10.1007/BF00239528. 
Figure 1
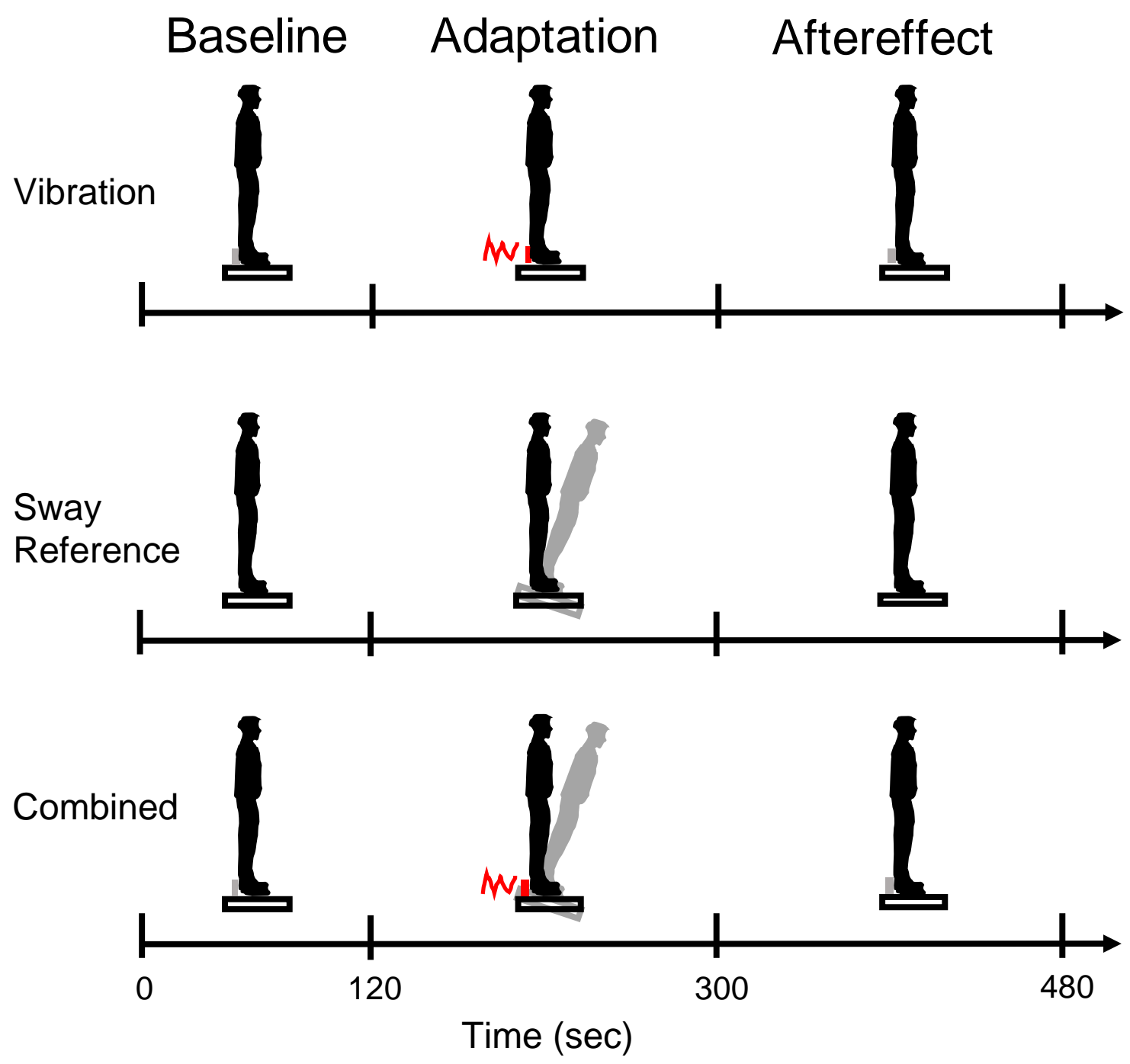

Figure 1. Schematic representation of the three conditions. 
Figure 2

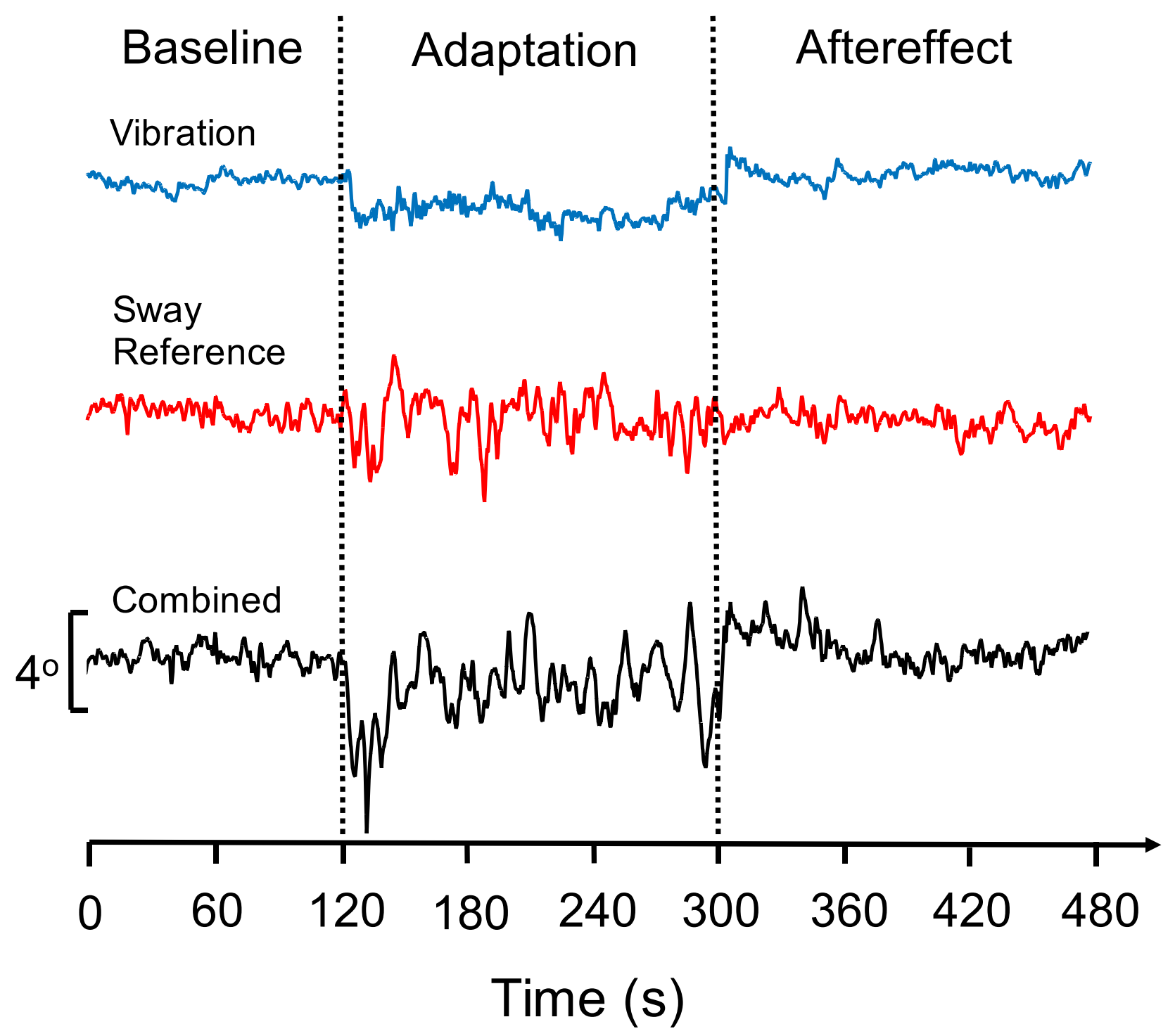

Figure 2. Sample traces of a representative participant's C7 tilt angle trajectory in the sagittal plane over time in the three conditions. 
Figure 3

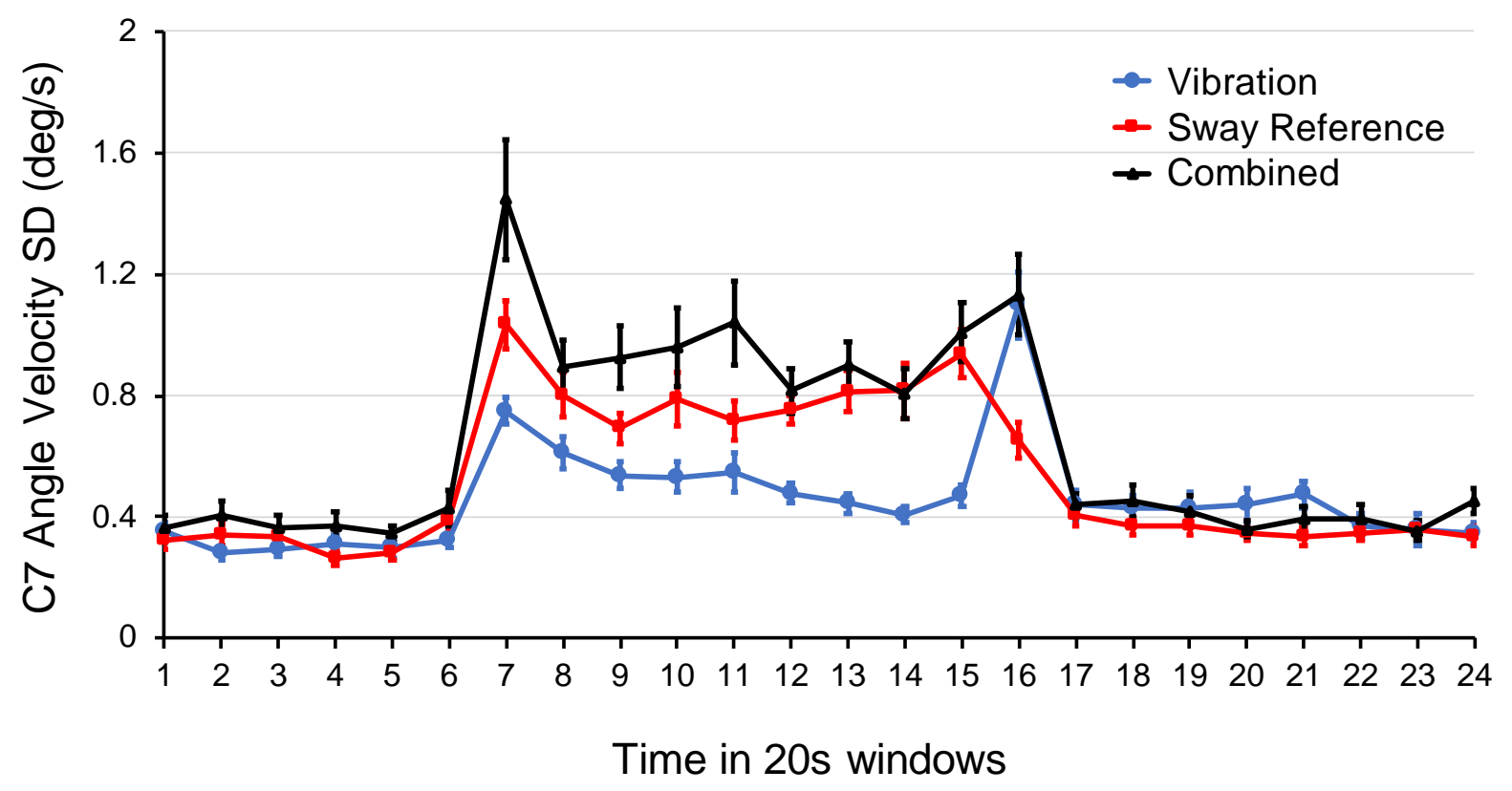

Figure 3. C7 tilt angle velocity Standard Deviation (SD), calculated over successive 20s windows throughout the $8 \mathrm{~min}$ trial for the three conditions. Baseline is shown in windows 1-6, Adaptation in windows 7-15 and Aftereffect in windows 16-24. Group mean and standard error $(n=17)$ is shown. 
Figure 4

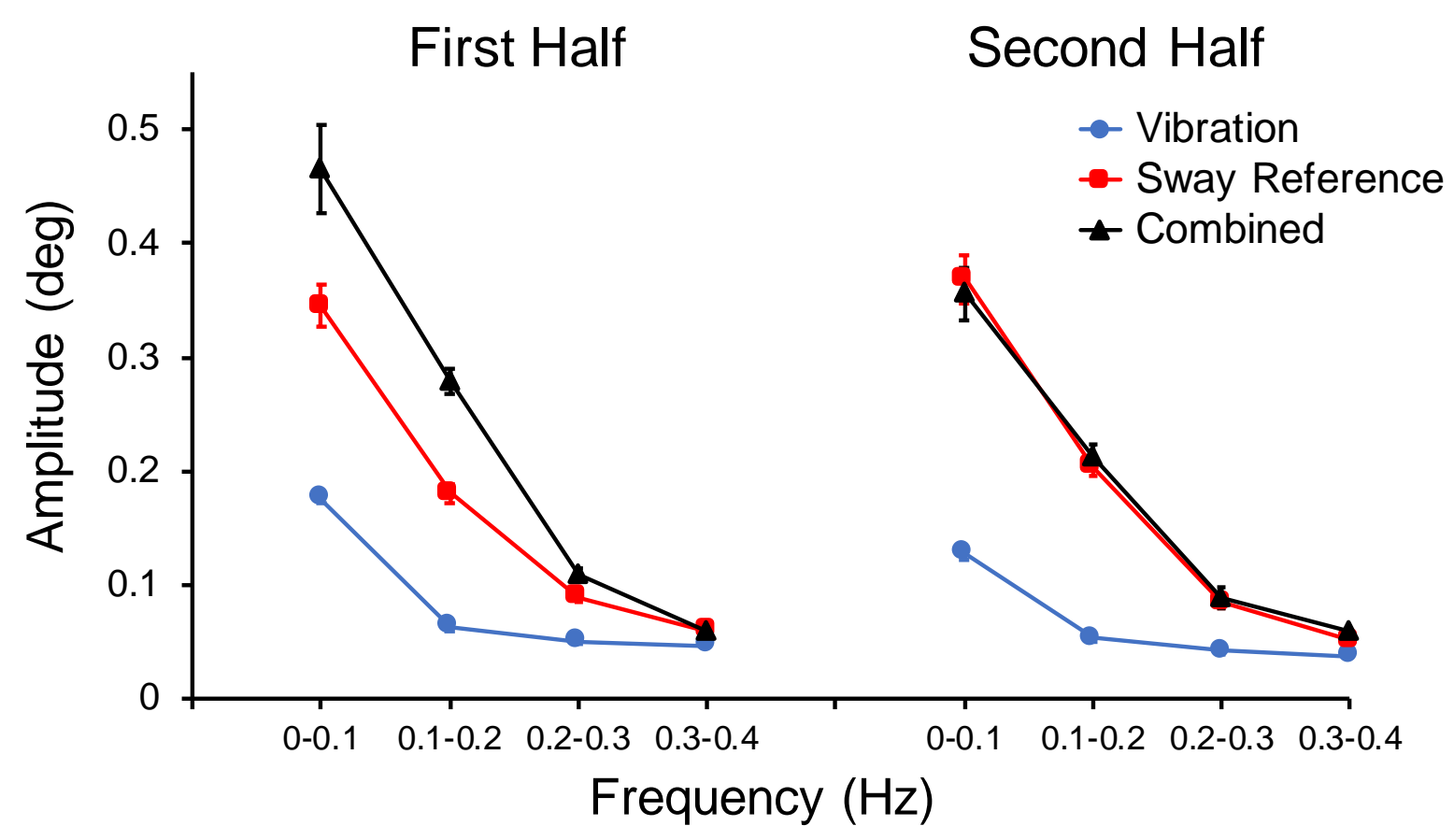

Figure 4. Spectral amplitude of C7 tilt angle trajectories plotted across 4 frequency bins in the first and second half of adaptation for the three conditions. Group mean and standard error $(n=17)$ is shown. Standard error bars are not visible in some of the data points due to their small size. 